\title{
Pervasive Decentralisation of Digital Infrastructures: A Framework for Blockchain enabled System and Use Case Analysis
}

\author{
Florian Glaser \\ Goethe University Frankfurt \\ fglaser@wiwi.uni-frankfurt.de
}

\begin{abstract}
Technological innovation and consequential decentralisation are driving forces in the ongoing evolution and increasing openness of digital infrastructures and services. One of the most discussed and allegedly disruptive innovations is the distributed database technology referred to as blockchain. Although it is still in its technological infancy, experimental adoption and customization seem to be in full progress in various potential fields of application ranging from decentralized grids for computation and storage to global financial services. However, the technology and its path of development still entail a lot of common unknowns for practitioners and researchers alike. Especially regarding the question how the technology could amend or be incorporated into the existing landscape of digital services, processes and infrastructures. Hence, in this article we develop an ontology that (1) clearly delineates common terminology, core concepts and components, their relationships as well as innovative features of blockchain technology. It further (2) connects these insights with implications for relevant types of digital market models. Our framework is of high theoretical and practical value as it provides researchers and practitioners a common basis for communication and means for guided analysis of blockchain applicability.
\end{abstract}

\section{Introduction}

Identification and valid analysis of blockchain ecosystems and application scenarios impose a prevailing issue for practitioners and researchers.

Despite many discussions, press releases and talks about blockchain technology, few truly and fully understand or can actually describe with certainty the basic or innovative features introduced by blockchain technology. This most likely also holds true for crossdisciplinary researchers from non-technical disciplines. Reasons might be the complex interplay of blockchain components and resulting properties that are hard to grasp in detail as well as the lack of a solid common knowledge base, especially in information systems (IS). The most recent publicly available blockchain instantiations, e.g. Ethereum, comprise a decentralized peer-to-peer network, a built-in public-key infrastructure, cryptographically enabled data structures for storing transactions as well as data attached to transactions and include a Turing complete programming language based on a replicated virtual machine. Each of these components can be itself quite complex to understand, especially for business, economic, social and political science researchers and practitioners.

Additionally, blockchain systems introduce new ways of decentralisation and delegation of services into the hands of autonomous interacting pieces of code, also referred to as smart contracts. These autonomous and hence trust-free setups also attack current trust establishing institutions and intermediaries, such as banks or market place operators. Incumbent role and business models in digital ecosystems might no longer apply in the context of blockchain systems. These circumstances lead to confusion and uncertainty regarding actual use cases and their technological and economical validity.

It comes with no surprise that an established and often cited phrase related to blockchain technology states that "blockchain is an innovative technology in search of use cases".

Nonetheless, potential use cases and their implications are frequently discussed by a variety of researchers, practitioners and governmental institutions alike. The current focus of the discussion is primarily associated with financial market infrastructure and related services, e.g. payments and post-trading. The likely reason for this single focused attention and media coverage is the allegedly disruptive potential of the technology for the financial sector. This storyline is currently rephrased by nearly every major bank [36] or consulting company [4] as well as central banks [17] and financial market authorities [8]. Its potential impact is often compared to the impact the internet had on global interconnectedness in societal as well as business environments. 
However, the how and why regarding these use cases is often missing in the discussions or descriptions which are often limited to an abstract and opaque level.

From a practical perspective, the accelerating process of decentralisation of services in areas like retail payment services [5] and asset management can hence be seen to be only a precursor of uprising scenarios for services in supply chain management, insurance, digital knowledge management, e-business and e-commerce. These developments are further pushed by societal trends towards a networked society [10] and platform-mediated services. Clients become increasingly connected as highly available, reliable network infrastructure is common even for retail customers. Thus, the need for intermediaries providing solid and reliable service infrastructure while leveraging economies of scale is diminishing with an increasing global digitization and interconnectedness.

These environmental developments in combination with digital innovation in form of blockchain technology probably hold opportunities to create and integrate new services and business models into the existing digital economy. Digital innovation life cycles can be described by four phases as reviewed by [11]: discovery, development, diffusion, impact. Discovery of blockchain technology can be dated to 2015 when the majority of financial institutions publicly announced interest into the innovation. Given that there are almost daily announcements in the news media of prototypes and tests by companies from various industries, the development phase is probably reached. According to [11] the relevant managerial questions arising during theses phases are "What constitutes the digital innovation's core feature set?", "To what potential organizational uses can it be put?", "What complementary products and services are needed to flesh out the 'whole product solution'?", "what other elements (organizational, technological) comprise a sound innovation system incorporating the digital innovation?".

We summarise these relevant managerial questions by formulating the following research question: How can blockchain (eco)systems and associated roles be described and analysed in the context of digital economies?

Against the backdrop of the outlined situation, the overall goal of this paper is twofold. First, by providing a comprehensive conceptual framework we aim to support practitioners to evaluate use cases, new business models and roles in the existing, evolving and emerging blockchain ecosystems. Second, the framework provides cross-disciplinary researchers and practitioners with a structured knowledge base and a common vocabulary regarding terms, concepts and their relationships as well as roles that is in line with the blockchain development community domain. It provides an ubiquitous language for blockchain system contexts.

The remainder of this paper is organized as follows. Section two provides a brief overview of the background of the technology and fundamental definitions. This also entails a review of related literature on blockchain technology in information systems. In section three we explain our research approach and methodology. Section four comprises the development of the conceptual framework. Section five contains a critical review of our results before we conclude and provide an outlook for future research in section six.

\section{Background}

\subsection{Related Literature}

The attention to blockchain technology as well as Cryptocurrencies is still quite limited in the discipline of information systems. This is astonishing as practitioners, especially in the finance and insurance sector are relentlessly working on the identification of reasonable use cases and timely technological validation in form of prototypes based on different blockchain technologies [43].

Although the adoption has already begun, due to the fact that almost all systems are open source, adoption can be more complicated as only recently emphasized by [12].

A common limitation of previous work on blockchain technology is that it is either sticking to a mono-disciplinary perspective, e.g. cryptographic security [28], consensus algorithms [23], economics [3], or focuses specific cryptocurrencies which are only a single purpose instantiation of a blockchain system [20].

The truly innovative character of the technology, however, is its openness and technologically driven capability to pervade multiple vertical layers of digital ecosystem infrastructure. Pervasiveness can span the backend database, the business logic, up to the organisational layers due to smart contracts' capabilities in form of autonomous rule and process representation. However, it is quite difficult to capture all these different and complex layers into a single perspective. The design science approach of [2] provides a first glimpse at the potential economic implications of these systems.

Consequently, the focus of this study is to derive a conceptual framework that unifies blockchain 
concepts and their relationships to digital market models into a single framework. This way, it guides researchers and practitioners in their analyses of blockchain systems research and application. We focus on the general purpose of information systems research discipline by describing and analysing the interdependencies between IT artifacts and their interaction with socio-technical systems [18].

In the context of information systems literature, we follow the call of [37] who request "(1) deeper theoretical work on the notion of infrastructures, (2) new theoretical lenses to understand the paradoxical nature of change and control in digital infrastructures, and (3) better understanding of the ways in which infrastructural change shapes IT governance, IS development, and promotes new effects across all levels of analysis."

\section{Research Design}

Theorizing in IS provides a variety of paths to be followed as summarised by [13]. Descriptive theories, i.e. theories that state "what is", are needed when very little is known about the phenomenon in question. Ontologies provide a framework for structured knowledge representation $[19,16]$. They establish concepts and their relationships in a specific knowledge domain to facilitate communication and collaboration in the explicit context of that domain [15]. Different variations of this type of theory are classification schemata, conceptual frameworks and taxonomies [13].

We rely on the description of basic components of an ontology as declared by [39]. According to them an ontology necessarily includes a vocabulary of terms and specifications of their meaning, i.e. definitions. We aim to construct a semi-formal ontology as described by [39] which primarily consists of textual descriptions. It provides the basis to derive the influence of the blockchain induced pervasive decentralisation on different levels of the digital infrastructure as well as market models.

One example for this type of approach is the work of [20]. They outline potential impacts of cryptocurrencies on digital payment system platforms and services. In contrast, however, our study explores a broader, more general scope of digital market models in the context of blockchain. Hence, we achieve a higher generalisability of obtained results, i.e. a more general applicability, as our framework is not bound to a single service domain.

Our approach is divided into four consecutive steps. First, we delineate the core concepts and features of blockchain systems and split blockchain systems into two logical layers. Second, we induct implications for the digital infrastructure and the governance of blockchain systems as well as general implications of pervasive decentralisation. Third, we derive the impact on digital market models.

\section{Ontology Development}

The subsequent analyses and ontology development is primarily based on the most comprehensive resource available for smart contract systems, i.e. the documentation of the Ethereum blockchain. We rely on the yellow paper [42] which describes the technology in detail. For vocabulary and concept naming we rely on the github documentation (https://github.com/ethereum/wiki) and the Ethereum blog (https://blog.ethereum.org). Concepts of the ontology are formatted italic.

\subsection{Blockchain System Concepts and Relationships}

We start with the definitions of a common set of blockchain components and relationships as a first part of our blockchain ontology.

Basically, a blockchain is a distributed, transactional database. Globally distributed nodes are linked by a peer-to-peer (P2P) communication network with its own layer of protocol messages for node communication and peer discovery. Nodes identify each other by their IP address and users reference each other via their public key. The corresponding private key of a user is used to cryptographically sign messages and transactions $(T x)$. Put differently, he authorises them by cryptographically assuring that they represent his intention.

A node is a physical/virtual machine that communicates via TCP/IP and UDP with other nodes. In contrast, a user is only represented by a public key address and could theoretically login from any other node. This is possible as each of the nodes maintains a database of all historical, valid transactions that have been sent between the nodes of the network. Transactions are grouped into blocks. Every block references the previous block of transactions and hence a temporal ordering of transactions is achieved. Order of transactions within a block are determined by a randomly selected node in line with the applied consensus algorithm. Light client nodes are configured such that they only collect the hashes of blocks of transactions to safe disk space and make the services available on less performant devices like smartphones. 
Every blockchain's core functionality is to provide validated, immutable transactions, i.e. database updates, that are consistent between a large number of nodes in a global network. Blockchains use complex data structures (e.g. merkle trees or patricia merkle trees) to efficiently store all transactions in a way that the current state of the system inherently depends on all previous transactions. Thus, changes (e.g. manipulations) to historical transactions in a single node result in invalid states if one would recalculate the current state from all historical transactions. If a node then proposes its transactions which are identified as invalid by other nodes, the node proposing invalid transactions will be ignored by the other nodes in the system as it is inconsistent (i.e. it violates the protocol) with the other nodes. For the sake of brevity, we refer to [28, 42, 27] for a more detailed description of these data structures.

It is worth noting, that the meaning of the word transaction can be ambiguous in the context of a blockchain. On the one hand, a blockchain is a database and in this traditional context it can simply mean the update of data in the database. On the other hand, a blockchain often facilitates the transfer of tokens, where a transaction then refers to the transfer of tokens from one user to another user. Tokens are either inherent to the system or implemented in higher layer scripting or programming languages.

Most implementations feature a built-in scripting language which is able, in a very limited way, similar to stored procedures in traditional databases, to execute additional business logic triggered by a transaction. More recent generations of the technology, for example Ethereum and Hyperledger, extend the basic idea of a scripting language by integrating a fully fledged programming language executed by an internal virtual machine. These programming languages have also access to complex data types and data structures and even small, locally separated databases that can be used to store and retrieve data. The code is deployed into the blockchain database at every node and can be triggered by certain transaction events. This enables far more possibilities than transactions of tokens as cryptocurrencies were intended to do.

These pieces of code are often referred to as smart contracts. Although, the notion of a contract is somewhat misleading. The origin of that notion dates

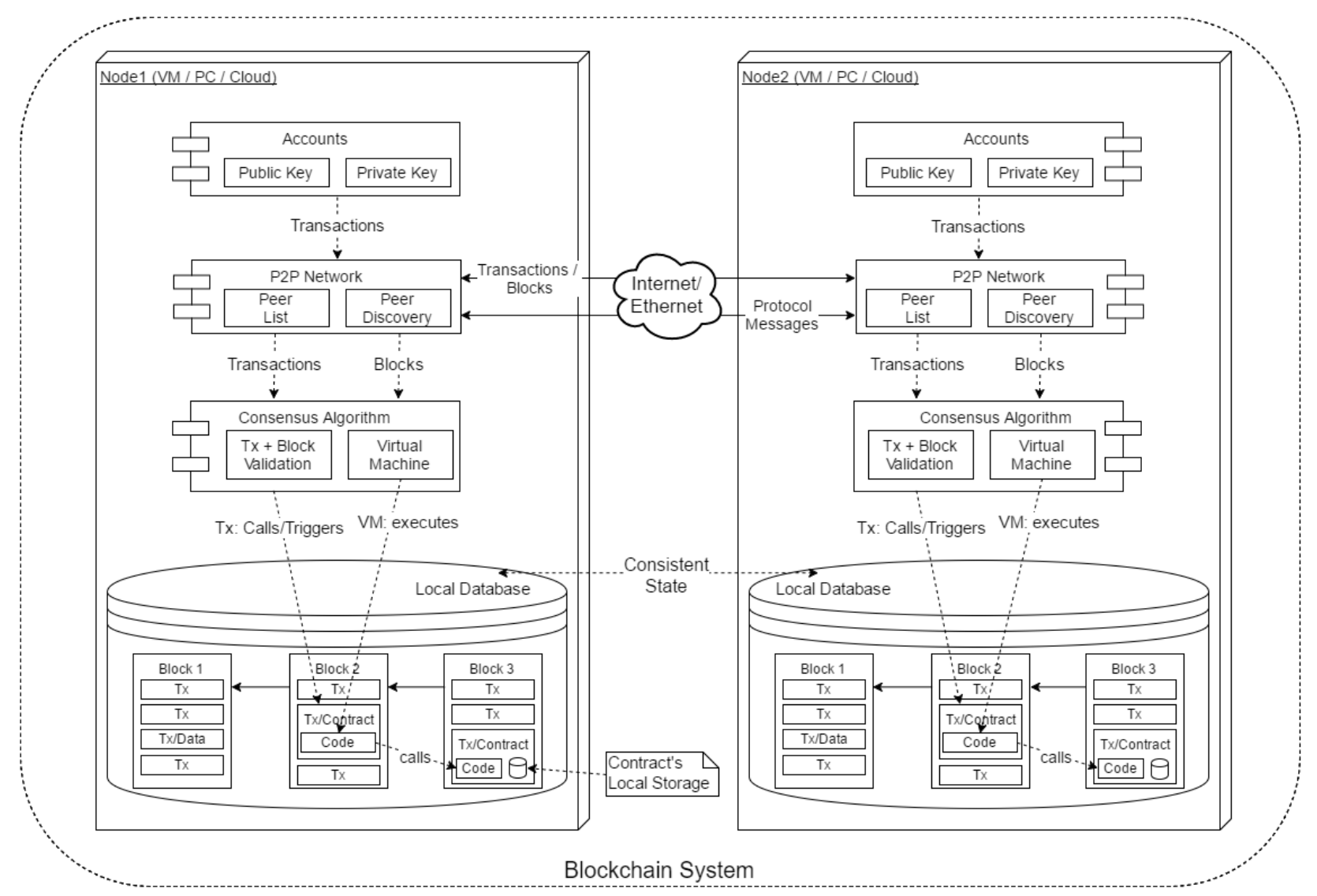

Figure 1. Blockchain System Concepts and Relaitionships 
back to the idea of code-based self-executing legal contracts as described by [34]. However, for purposes of consistency we keep the notion of smart contracts.

Smart contract execution can be compared to atomic transactions, i.e., the transition from the current database state to the next database state by changing data. If one part of the code execution fails, the whole transaction fails and the next state is not reached. Execution time is limited in an economical way, i.e. you have to pay for execution time. This prevents nodes from executing one smart contract infinitely. As the state transition, i.e., smart contract code execution is performed on every node in the network, infinite execution would put the whole blockchain system to halt. One consequence is that smart contracts cannot trigger themselves as this would require that they are continuously executed. There is no self-execution at a certain point in time or environmental event without explicit external intervention. This is a common misunderstanding of how these systems operate.

Blockchain systems currently face some other technical limitations: Capacity, latency and query capabilities are quite limited in comparison to other distributed database systems as described by [24]. However, these constraints are likely to be of transient nature and further improvements and future innovations are probably able to solve current limitations. For the sake of brevity we leave technical limitations aside during our analysis.

Figure 1 depicts the previous conceptual descriptions to provide a comprehensive overview of generic blockchain system components and relationships.

In the next two sections we review blockchain system infrastructure, define concepts and relations and derive implications for digital infrastructures. We then discuss the implications for digital market models.

\subsection{Pervasive Decentralisation of Digital Infrastructure}

We start with further dividing blockchain systems

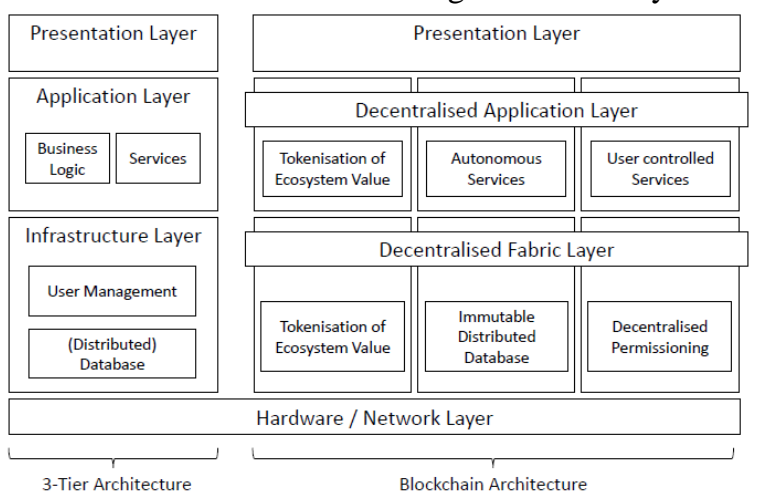

Figure 2. Layers of Blockchain Systems into two layers of code. The first layer is the so called fabric layer, which is a common concept in the global blockchain development community. The fabric layer denotes the actual blockchain code base that comprises the communication layer, the publickey infrastructure, the data structures to construct and maintain the database as well as the execution environment for smart contract languages. The second layer comprises the application logic of services implemented in form of smart contracts. We refer to the second layer as application layer. Services based on one or more smart contracts are commonly called Decentralised Applications (DApps). Figure 2 visualises the different layers.

A remarkable fact worth noting is, that the system spans across multiple layers of traditional n-Tier architectures. This is the reason for the technology's pervasiveness and has far reaching implications as we will discuss later.

The Fabric Layer. It is crucial to note, that there is a strong centralisation of control of the fabric layer. Whoever develops and maintains the fabric layer is in ultimate control of the whole system's functioning. Even if he cannot directly modify the state of the system as represented by the blockchain database.

If the fabric layer is developed by a single company and the code base is not open source, the whole system will always be in the hand of this company. If it is kept open source, the organization of developers and maintenance of the code is more complex. We refer to $[6,14,25,22]$ for background on open source communities. This is also something that needs to be considered in the context of software system adoption by corporations as is emphasized by [26].

The fabric layer fulfills basic services. Databases usually come with an integrated module to manage different types of users that can have different levels of permissions. For example, a user might only be able to read certain subsets of the database and is not allowed to send any transactions, i.e., changes to the data. A super user, however, usually has the full set of rights or can grant them. Blockchain systems, so far, make no differentiation between users and user management modules only provide rudimentary functionality like account creation and basic password management.

This also implies that every user has full transparency regarding the transactions and deployed smart contract code of other blockchain system users.

In a setup, however, where only a set of permissioned users can see and validate transactions and blocks, privacy is no longer an issue if permissioned parties would see the content in a traditional setup as well. This permissioned blockchain system or private blockchain is in stark 
contrast to the idea of public blockchains like Bitcoin or Ethereum. However, for testing purposes and business setups it is the primary choice.

Hybrid systems are currently discussed as well, however, at the time of writing there is no public hybrid system live and running. Ripple could be considered a hybrid system, although it does not maintain a blockchain of transactions in the fashion we described. From an economic perspective, a private permissioned system merely resembles an intra- or inter-group technology upgrade. If there is no trust issue among nodes with respect to validity of database updates the only reason to opt for a blockchain is its immutable $\log$ of historical transactions for audibility purposes.

In an economic context, a hybrid system enables a restricted set of users to access the services. Hence a hybrid blockchain can be considered a club good. Users are excludable from the system but the admitted users have no further restrictions with respect to usage of the systems services.

A public blockchain, on the contrary, resembles a public good. That is, users are non-excludable from its services and there is no rivalry among users. If there is rivalry for some reason, it could be considered a common good. If public blockchains enable basic services like car or citizenship registry, the government would be responsible for provision and maintenance of fabric layer. Provision of public goods is a complex undertaking [40] people building the system might get no rewards besides the system itself and others may use it for free without contributing. It can be incentivised by so called dominant assurance contracts as proposed by [35]. The literature regarding the economics of goods is actually quite vast. We refer to [21] for an overview. Besides, a completely open setup, where any user can join if he can install the fabric layer software, introduces potential issues regarding regulations of service provision. For example, in the case of a payment service, anti money-laundering and know your customer processes are required by regulation and hinder adoption, especially in the financial sector $[8,9]$.

The Application Layer. So far we discussed the levels of decentralisation of the fabric layer which is comprising the blockchain system itself. We will now discuss the implications of decentralisation of the application layer in more detail.

The blockchain itself is developed by a group of architects and developers and hence is always under control of these development teams.

On the contrary, the code of the application layer can be written and bound to the system by any participant. The code itself is then under control of the participant who deployed the piece of code. It follows, that the control at the application layer is distributed among the participants who deployed the code.

Once the system is running, open to the public and users start deploying their own code onto the blockchain system, the developers of the fabric layer are no longer in control of what is actually happening on top of the system, i.e. within the application layer.

Consequently, the control of the application layer is pushed into the hands of a decentralised user space and its control is equally decentralised. The code of smart contracts, can be built in such a way, that the control is entirely left over to the piece of code deployed. If a transaction contains such smart contract code, a new participant (address) is registered in the system. The new participant is the smart contract and has its own address in form of a public address. If there is no access control mechanism implemented by the participant who deployed the smart contract, the code becomes autonomous. This leads to a setup where only the piece of code itself 'determines' what happens when it is triggered, based on the programmed rules it contains. Hence, participants can hand over decision making to an autonomously deciding piece of software that is accessible for other participants of the system.

The resulting decentralisation of control in combination with the immutable representation of transfer of possession or, more generally, speaking the transition of system states, leads to the common notion of 'trustless systems'. These can be build on the application layer.

An immediate consequence is that business logic based on smart contracts can use the blockchain system with all its features in an autonomous way. Put differently, smart contracts can represent the business logic, e.g. market mechanisms or decision making and in addition communicate with each other, i.e., call or trigger each other. They can hence create a web of tiny services that enable the creation and autonomous activity of very complex systems. However, recall that the activity must always be triggered by an external impulse. A smart contract could, for example, realise a service that maintains a small database accessible only by this contract. It could evaluate data attached to a transaction and trigger further transactions to other users or smart contracts. Hence, smart contracts could implement autonomous market mechanisms or complex microservice interactions which, in total, realise more sophisticated service logic like an autonomous portfolio management service. In summary, autonomous service provision in combination with 
trustless setups of smart contracts could replace trust intermediaries.

It is worth noting here, that any information that is not generated by a transaction has to be introduced as data attached to a transaction. This also holds for time events. Put differently, any activity in the system needs to be triggered by a node controlled from outside of the network (which of course can be another software system). This is a functional limitation to be kept in mind.

The basic set of blockchain functionalities enables users to transact whatever is represented by the builtin tokens of the blockchain system. However, tokens can also be represented by a smart contract as a mapping of user addresses to an account balance stored in the smart contract. That is, when the smart contract transfers any amount of the token to a another it does so by setting an entry into its local database which represents the amount and the user address. Note that this is equivalent to what a bank does if it credits a certain amount of a currency to a customer's account. Consequently, these tokens could also be maintained autonomously by the smart contract.

However, merely the technical creation of the token does not attach any value to it. The platform openness of public blockchains influences this dimension of value representation in blockchain based ecosystems as we discuss next. If an ecosystem, comprised of the services interacting on the application layer, is self-sufficient in an economical sense of value transaction, it can be considered self-contained or closed. That is, if services in the system only rely upon other services or information generated within the system. We provide an example for such a system setup in the context of the internet of things. Assume that spare parts which are delivered by autonomous drones can be paid with the electricity produced by the spare part purchaser's energy plants. These services could interact without exchanging with other ecosystems as they are directly offsetting each other. If this is the case, value, or valuable services are created within the system and can also be redeemed or spent within the system. Such a closedness of the ecosystem implies that there is no need to bind to external systems in order to exchange information or tokens of any value. Users and services can rely on internally issued tokens in order to interoperate, i.e. use the token as a medium for exchange. An extreme scenario would be that a whole economy is represented on a blockchain system. for example, the ecosystem of an online multiplayer game. It follows that the higher the closedness of the ecosystem, the more suitable is a blockchain infrastructure.
The other case would be that frequent interaction with external services is needed in order to bind any value to the system. In that case, the decentralisation of control ends at the boundaries to other systems and the full potential of autonomous smart contracts can no longer be leveraged. The binding interoperation then introduces trust into the external institution and the interface(s) the system needs to be bound to. For example, if we build a payment system and there is no way to purchase goods or services with the internal token, then we need an exchange to transfer the token into a valuable currency. We then have a trusted interface to the issuer of the outside currency to be valuable and stable in value.

Furthermore, the actual types of services, and the market mechanisms (provided on the application layer) by which these services are priced determine the relevant roles in such an ecosystem. The major difference to current digital market models is, however, the possible decentralisation of intermediaries by autonomous DApps. Hence, fundamental question becomes whether the traditional roles of digital market models can be delegated to an autonomous piece of code. For an overview of roles and market models in the context of digital platforms we refer to [38] and will discuss the implications in the next section.

Previous considerations also introduce collaboration on different levels, either in form of standardization of smart contract interfaces or the standardization of complete services and process interoperability. For incumbent digital services and standardization we refer to [30]. However, the design of digital services itself can be quite sophisticated

Table 1. Applicability Analysis Framework

\begin{tabular}{|c|c|c|}
\hline Layer & Dimension & Considerations \\
\hline 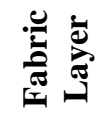 & $\begin{array}{c}\text { Governance } \\
\text { Type }\end{array}$ & $\begin{array}{ll}\text { - } & \text { Club Good } \\
\text { - } & \text { Common Good } \\
\text { - } & \text { Public Good }\end{array}$ \\
\hline \multirow{3}{*}{ 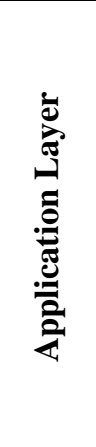 } & $\begin{array}{l}\text { Ecosystem } \\
\text { Closedness }\end{array}$ & $\begin{array}{ll}\text { - } & \text { Self-Contained } \\
\text { - } & \text { Trusted Interfaces } \\
\end{array}$ \\
\hline & Value Linking & $\begin{array}{ll}\text { - } & \text { Community } \\
& \text { Currency } \\
\text { - } & \text { Debt/Equity } \\
& \text { Issuance } \\
\text { - } & \text { Commercial Bank } \\
\text { - } & \text { Central Bank } \\
\end{array}$ \\
\hline & Market Type & $\begin{array}{ll}\text { - } & \text { Multi-sided } \\
\text { - } & \text { Collaborative } \\
\text { - } & \text { P2P }\end{array}$ \\
\hline
\end{tabular}

and several dimensions have to be taken into account according to the findings of [41]. 
These considerations further lead to the known critical mass dilemma in a multi-sided market [32] if the adoption by either service providers or customers is low. Both, customers as well as services providers are needed simultaneously in order to achieve a critical mass and subsequently a high level of closedness of the system.

The discussion so far is also tightly related to the real-world problem of how currency actually becomes valuable. This problem is usually solved by trust in a system of central banks as well as commercial banks. However, other ideas have been around for several decades, e.g. free banking systems where the same currency varies in value dependent on the bank that issued the note or coin [33]. Another possibility is a community currency which resembles to the setup of a closed ecosystem.

The traditional ways of binding an ecosystem to valuable tokens, i.e., database entries are commercial bank money and central bank money issuance. A fundamental game changer for a blockchain system emerges if a bank directly provides value backing of blockchain issued tokens, e.g. by issuing commercial bank money on the blockchain. The closedness of the system becomes irrelevant, as one can use the commercial money token as medium to the outside world. It does not make any difference if the token is the inherent token of the respective blockchain system or a token that is issued by a smart contract controlled by the bank. This setup would practically solve the problem of ecosystem or value binding with traditional means. However, the needed trust in institutions is, then again, necessary at the trusted interfaces.

Another possibility of ecosystem value linking would be a central bank backing of one or more tokens of the system. This would leverage the position of a central bank, which is coupled directly to the economy of one or more nations. Although this would facilitate the diffusion and ease of use of blockchain systems, it would, once again, introduce the hierarchies and links to institutional trust of the current financial system. The question boils down to whether a single central governmental authority or multiple private institutions are the preferable option if community currency or ecosystem closedness are not applicable. Actual discussions by central banks regarding digital currency issuance became public only recently $[7,31]$.

\subsection{Implications for Digital Platforms and Market Models}

Blockchain technology is by design a multi user system. It is designed for continuous, non-centrally governed interaction among (large) heterogeneous groups of participants. Furthermore, it supports the independent development and deployment of autonomous, collaborative and highly interoperable services by every user of the system. Against the backdrop of these core capabilities we consequentially narrow our perspective on digital market models to those likely to provide valid use cases for blockchains. A market model should rely upon these kinds of participant groups and also interaction among them. We have identified three types of market models that match these criteria, namely multi-sided, collaborative and P2P markets [1].

Multi-sided markets are characterised by the interaction of multiple parties with different interests in a single market mechanism [29]. Usually, intermediaries provide services of information or product brokerage in the role of platform providers. Examples are credit cards and stock exchanges. The role of intermediaries is at stake in blokchain based ecosystems. They are subject to decentralisation by autonomous DApps if the service is of transactional character and of low complexity.

Collaborative markets are equally characterised by multiple parties interacting on platforms which provide basic means of exchange for information or goods. Examples are open source software or open knowledge (e.g. wikipedia) platforms. As these types of platforms or markets are already open and often closed ecosystems they provide suitable candidates to profit from blockchain infrastructure. As payments for contributions are an issue that could be solved by blockchain infrastructure.

P2P markets are natural candidates to profit from blockchain infrastructure due to their high ecosystem closedness. Blockchain systems could provide the infrastructure to decentralise intermediary services and means of trustless payments in comparison to current systems. Examples are Foodsharing, Couchsurfing, P2P-Lending and Filesharing.

The last artifact of our ontology is table 1 and summarises the four dimensions that should be covered during a blockchain applicability analysis. It can be applied by reproducing our considerations in the last two sections in an explicit business domain.

\section{Discussion}

Our framework development has shown that there are two imminent implications from an economic perspective. First, the platform providers underlying all market models could be replaced by decentralised blockchain systems. Especially if their services are neither highly complex nor computational intensive. 
For example crowdfunding, or crowdinvesting platform providers in collaborative markets and platform providers in multi-sided markets, e.g. Uber or AirBnb.

Second, trusted third-party service providers replacement in multi-sided markets might be limited fit due to the governance centralisation of the fabric layer. Additional conditions must be considered to make it an attractive alternative to a central thirdparty. The economic incentives of the fabric layer governance party must be in line with the application layer party. This relationship should also be rather stable over time in order to ensure long term support for the ecosystem. This argumentation is not limited to any market model. Decentralisation and hence trust decentralisation is only as good as the most basic layer - the fabric layer.

With our ontology we contribute to theory in general by introducing an ontology for the domain of blockchain systems. The framework and its development process provide in-depth knowledge on blockchain systems in general and their influence on the digital ecosystems in particular. We further contribute to the literature on strategic frameworks that identify new sources of value creation in form of digital product platforms [44]. The framework is also of high practical relevance due to its guiding capabilities for the analysis of blockchain use cases.

\section{Conclusion and Outlook}

The initially stated goal of this work is to derive a framework that (1) provides a common basis of concepts for the domain of blockchain systems and (2) can be applied by researchers and practitioners to assess the implications of Blockchain technology in various economic and academic contexts. In this work we develop an ontology that describes common components of blockchain systems and proposes a common vocabulary for communication. We further introduce the perspective of pervasive decentralisation of multiple layers of digital infrastructure by blockchain technology. Finally, we outline implications for market models in the digital economy, that are likely to be affected by blockchain systems.

Interoperability is key in any e-Business environment. Blockchain infrastructure has the potential to contribute due to its pervasive, decentralised and open design. Future research could hence focus on how blockchain systems can be leveraged for better interoperability in electronic markets.

\section{References}

[1] Avital, M., M. Andersson, J. Nickerson, Arun Sundararajan, Marshall Van Alstyne, and D. Verhoeven, "The Collaborative Economy: A Disruptive Innovation or Much Ado about Nothing?", in Thirty-Fifth International Conference on Information Systems, Auckland. 2014.

[2] Beck, R., J. Stenum Czepluch, N. Lollike, and S. Malone, "Blockchain - The Gateway to Trust-free Cryptographic Transactions", in Twent-Fourth European Conference on Information Systems (ECIS). 2016: Istanbul, Turkey.

[3] Brandvold, M., P. Molnár, K. Vagstad, and Andreas Valstad, Ole Christian, "Price discovery on Bitcoin exchanges", Journal of International Financial Markets, Institutions and Money, 36, 2015, pp. 18-35.

[4] Buehler, K., D. Chiarella, H. Heidegger, M. Lemerle, A. Lal, and J. Moon, "Beyond the Hype: Blockchains in Capital Markets", 2015. https://www.weusecoins.com/assets/pdf/library/McKinsey \%20Blockchains\%20in\%20Capital\%20Markets_2015.pdf, accessed: 10.06.2016.

[5] Carminati, B., E. Ferrari, and N.H. Tran, "Trustworthy and effective person-to-person payments over multi-hop MANETs", Journal of Network and Computer Applications, 60, 2016, pp. 1-18.

[6] Cheliotis, G., "From open source to open content: Organization, licensing and decision processes in open cultural production", Decision Support Systems, 47(3), 2009, pp. 229-244.

[7] Danezis, G. and S. Meiklejohn, "Centrally Banked Cryptocurrencies", 2016. http://www0.cs.ucl.ac.uk/staff/G.Danezis/papers/ndss16cur rencies.pdf.

[8] ESMA, "The Distributed Ledger Technology Applied to Securities Markets", 2016. www.esma.europa.eu, accessed: 10.06.2016.

[9] European Central Bank, Virtual currency schemes, European Central Bank, Frankfurt-on-Main, 2012.

[10] Faltings, B., K. Leyton-Brown, and P. Ipeirotis, EC'12: Proceedings of the 13th ACM Conference on Electronic Commerce : June 4-8, 2012, Valencia, Spain, ACM, New York, 2012.

[11] Fichman, R.G., B.L. Dos Santos, and Z. Zheng, "Digital Innovation as a fundamental and powerful Concept in the Information Systems Curriculum", MIS Quarterly, 38(2), 2014, pp. 329-353.

[12] Gallego, M.D., P. Luna, and S. Bueno, "User acceptance model of open source software", Computers in Human Behavior, 24(5), 2008, pp. 2199-2216.

[13] Gregor, S., "The Nature of Theory In Information Systems", MIS Quarterly, 30(3), 2006, pp. 611-642.

[14] Grewal, R., G.L. Lilien, and G. Mallapragada, "Location, Location, Location: How Network Embeddedness Affects Project Success in Open Source Systems", Management Science, 52(7), 2006, pp. 10431056. 
[15] Gruber, T.R., "A translation approach to portable ontology specifications", Knowledge Acquisition, 5(2), 1993, pp. 199-220.

[16] Guarino, N., "Formal ontology, conceptual analysis and knowledge representation", 43, 1995, pp. 625-640.

[17] Haldane, A., "Old Money, New Money", 2015. http://www.bankofengland.co.uk/research/Documents/conf erences/ah0515.pdf, accessed: 15.06.2016.

[18] Jones, M.R. and H. Karsten, "Giddens's Structuration Theory and Information Systems Research", MIS Quarterly, 32(1), 2008, pp. 127-157.

[19] Jurisica, I., J. Mylopoulos, and E. Yu, "Ontologies for Knowledge Management: An Information Systems Perspective", Knowledge and Information Systems, 6(4), 2004, pp. 380-401.

[20] Kazan, E., C.-W. Tan, and E.T. Lim, "Towards a Framework of Digital Platform Disruption: A Comparative Study of Centralized \& Decentralized Digital Payment Providers", in 25th Australasian Conference on Information Systems. 2014.

[21] Ledyard, J., Public goods: A survey of experimental research, David K. Levine, 1997.

[22] Lindman, J., T. Kinnari, and M. Rossi, "Business Roles in the Emerging Open-Data Ecosystem", IEEE Software, 2015, pp. 1-7.

[23] Luu, L., J. Teutsch, R. Kulkarni, and P. Saxena, "Demystifying Incentives in the Consensus Computer", in Proceedings of the 22Nd ACM SIGSAC Conference on Computer and Communications Security. 2015. ACM: New York, NY, USA.

[24] McConaghy, T., R. Marques, A. Müller, D. de Jonghe, T. McConaghy, G. McMullen, R. Hendersen, S. Bellemare, and A. Granzotto, "BigchainDB: A Scalable Blockchain Database", 2016. https://www.bigchaindb.com/whitepaper/, accessed: 12.06.2016.

[25] Midha, V. and P. Palvia, "Factors affecting the success of Open Source Software", Journal of Systems and Software, 85(4), 2012, pp. 895-905.

[26] Murphy, S. and S. Cox, "Classifying Organizational Adoption of Open Source Software: A Proposal", in Open Source Systems: Integrating Communities, K. Crowston, I. Hammouda, B. Lundell, G. Robles, J. Gamalielsson, and J. Lindman, Editors. 2016. Springer International Publishing: Cham.

[27] Nakamoto, S., "Bitcoin: A Peer-to-Peer Electronic Cash System", 2008. http://bitcoin.org/bitcoin.pdf, accessed: 06.12.2013.

[28] Narayanan, A., J. Bonneau, and E. Felten, "Bitcoin and Cryptocurrency Technologies", 2016. https://www.coursera.org/course/bitcointech, accessed: 14.06.2016.

[29] Parker, G.G. and M.W. van Alstyne, "Two-Sided Network Effects: A Theory of Information Product
Design", Management Science, 51(10), 2005, pp. 14941504.

[30] Rai, A. and X. Tang, "Research Commentary Information Technology-Enabled Business Models: A Conceptual Framework and a Coevolution Perspective for Future Research", Information Systems Research, 25(1), 2014, pp. 1-14.

[31] Raskin, M. and D. Yermack, Digital Currencies, Decentralized Ledgers, and the Future of Central Banking.

[32] Rochet, J.-C. and J. Tirole, "Two-sided markets: a progress report", RAND Journal of Economics, 37(3), 2006, pp. 645-667.

[33] Rolnick, A.J. and W.E. Weber, "New Evidence on the Free Banking Era", The American Economic Review, 73(5), 1983, pp. 1080-1091.

[34] Szabo, N., "Formalizing and Securing Relationships on Public Networks", 1997.

[35] Tabarrok, A., "The private provision of public goods via dominant assurance contracts", Public Choice, 96, 1998, pp. 345-362.

[36] Taylor, S., "Blockchain: understanding the potential", 2015.

https://www.barclayscorporate.com/content/dam/corppubli c/corporate/Documents/insight/blockchain_understanding_t he_potential.pdf.

[37] Tilson, D., K. Lyytinen, and C. Sørensen, "Research Commentary -Digital Infrastructures: The Missing IS Research Agenda", Information Systems Research, 21(4), 2010, pp. 748-759.

[38] Tuunainen, V.K. and T. Tuunanen, "IISIn - A Model for Analyzing ICT Intensive Service Innovations in n-Sided Markets", in 2011 44th Hawaii International Conference on System Sciences (HICSS 2011), Kauai, HI. 2011.

[39] Uschold, M. and M. Gruninger, "Ontologies: principles, methods and applications", The Knowledge Engineering Review, 11(2), 1996, pp. 93-136.

[40] Varian, H.R., "Sequential contributions to public goods", Journal of Public Economics, 53, 1994, pp. 165186.

[41] Williams, K., S. Chatterjee, and M. Rossi, "Design of emerging digital services: A taxonomy", European Journal of Information Systems, 17(S5), 2008, pp. 505-517.

[42] Wood, G., "Ethereum: A secure decentralised generalised transaction ledger", 2014. http://gavwood.com/paper.pdf, accessed: 14.06.2016.

[43] World Economic Forum, "The future of financial infrastructure", 2016. http://www3.weforum.org/docs/WEF_The_future_of_finan cial_infrastructure.pdf, accessed: 25.08.2016.

[44] Yoo, Y., O. Henfridsson, and K. Lyytinen, "Research Commentary - The New Organizing Logic of Digital Innovation: An Agenda for Information Systems Research", Information Systems Research, 21(4), 2010, pp. 724-735. 